\title{
Evaluation of (Fludora® Fusion) (A Mixture of Deltamethrin and Clothianidin) For Indoor Residual Spraying Against Insecticide Resistant Strain of Anopheles Stephensi in A Malarious Area in Bandar Abbas , Iran
}

Hassan Vatandoost ( $\square$ hvatandoost1@yahoo.com )

Tehran University of Medical Sciences https://orcid.org/0000-0002-5983-9420

Fatemeh Nikpoor

Tehran University of Medical Sciences

Alireza Sanei Dehkordi

Hormozgan University of Medical Sciences

Mohammad Mussa Soleimani

Hormozgan University of Medical Sciences

abdolhossein madani

Hormozgan University of Medical Sciences

AR Mojahedi

Hormozgan University of Medical Sciences

M Yarian

Hormozgan University of Medical Sciences

Ahmad Raeisi

Tehran University of Medical Sciences

\section{Research}

Keywords: Fludora ${ }^{\circledR}$ Fusion, An.stephensi, residual, malaria, clothianidin

Posted Date: July 20th, 2021

DOI: https://doi.org/10.21203/rs.3.rs-697465/v1

License: (c) (i) This work is licensed under a Creative Commons Attribution 4.0 International License.

Read Full License 


\section{Abstract}

Objetives: Malaria is a health problems in the world. WHO recomended several insecticides for Indoor residual spraying. Due to continious use of insecticides, the malaria vectors show resistant to different groups of insecticides. The aim of study was to evaluate the new mixture of pesticides Fludora ${ }^{\circledR}$ Fusion) (a mixture of deltamethrin and clothianidin) for indoor residual spraying against insecticide resistant strain of Anopheles stephensi in a malarious area in Bandar Abbas, Iran.

Materials and Methods: The residual effect of Fludora ${ }^{\circledR}$ fusion (WP-SB $562.5 \mathrm{~g} / \mathrm{kg}$ in $100 \mathrm{~g} \mathrm{wsb}$ ) was evauated on various local surfaces of rooms such as mud and plaster as well as thatch roofs and wooden. Wordl Health Organization (WHO) standard cones using contact bioassays were carried out using laboratory reared, carbamate and pyrethroid resistant strain of Anopheles stephensi . Contact bioassays were carried out on sprayed surfaces. The cut of point of WHO is $80 \%$ mortality. Relative humidity and temperature of the test rooms has been recorded during the bioassay experiments

Results: Contact bioassay tests was carried out on days 1, 5,15,30, 60, 90, 120,150, 180,210,240,290 days after application. The mortality in all surfaces until 8 months is more than $80 \%$. Mortality rate after 9 months on plaster and mud was more than $80 \%$. The results form cement and wood was less than $80 \%$.

Discussion and conclusión: Indoor residual spraying with Fludora ${ }^{\circledR}$ Fusion induced high and prolonged mortality of carbamate and pyrethroid-resistant malaria vectors for 9 months mostly due to the clothianidin component. This mixture is an important Integrated Vector Management for control of resistant malaria vector.

\section{Introduction}

According to the current WHO report (WHO, 2020) (1), malaria continues to have a devastating impact on people's health and livelihoods around the world. According to the latest available data, about 3.2 billion people were at risk of the disease in 97 countries, territories and areas in 2013, and an estimated 198 million cases occurred (range: 124 million-283 million). In the same year, the disease killed about 584000 people, mostly children aged under 5 years in sub-Saharan Africa. In most countries where malaria is endemic, the disease disproportionately affects poor and disadvantaged people, who have limited access to health facilities and can barely afford the recommended treatment. Timely and affordable access to effective vector control interventions by populations that need them, including those to mitigate insecticide resistance and residual transmission, is a key component of malaria control and elimination efforts. Numerous interventions are under development aimed at addressing these areas. These include new insecticides, formulations or methods of application, new attractants and repellents, new bioactive agents (e.g., fungi or endo-symbionts), new mosquito life-cycle targets (e.g. sugar feeding, mating or oviposition phases), genetically modified mosquitoes, and endectocides.. WHO recommended insecticides for indoor residual spraying against malaria vectors are: DDT ,Malathion, Fenitrothion, Pirimiphos-methyl, Bendiocarb, Propoxur, Alpha-cypermethrin, Bifenthrin, Cyfluthrin, Deltamethrin 
,Etofenprox, Lambda-cyhalothrin, Clothianidin (WHO, 2018) (2). Most of which were short-lived on home wall substrates (2-5 months) thus requiring multiple resource-demanding IRS campaign rounds when used in areas with stable malaria transmission. Malaria vectors have also developed resistance to these conventional insecticides which is now widespread and increasing in intensity across Africa [6] and this, together with their short residual effect is driving the development of a new generation of long-lasting IRS insecticides to which local vectors are largely susceptible.The neonicotinoid, clothianidin is a new repurposed insecticide which was recently added to the WHO's list of pre-qualified insecticides for use in indoor residual spraying [3]. Clothianidin presents a new mode action which differs from that of conventional public health insecticides acting as an agonist on nicotinic acetylcholine receptors (nAChR) [4]. Owing to its novel mode of action, it shows potential to provide improved control of vector populations that have developed resistance to older public health insecticides. The addition of clothianidin to the portfolio of IRS insecticides also provides an opportunity to mitigate the development and spread of insecticide resistance in malaria vectors through the rotational use of IRS insecticides and the development of mixture IRS co-formulations [2]. Insecticide mixtures for IRS need to be explored with new public health insecticides when they become available because mixtures have the dual potential to improve malaria vector control through the combined effect of both active ingredients and contribute to insecticide resistance management, especially in areas where resistance to both active ingredients is not yet established [5]. In a previous experimental hut study in Benin, a tank mix of clothianidin and deltamethrin induced high and prolonged mortality (8-9 months) in wild pyrethroid resistant An. gambiae (s.l.) owing to the clothianidin component and early exiting of mosquitoes from experimental huts due to the pyrethroid component [6].Currently, 8 registered neonicotinoid insecticides are available commercially worldwide [WHO 2020,b) (7)

\section{Material And Methods}

Study area; Malarious area of Bandar Abbas southern Iran was selected for experiments (Fig.1)

\section{Materials:}

The mixture of two insecticide, chlothianidin and deltamethrin (Fludora ${ }^{\circledR}$ Fusion) is provided by Bayer company. Fludora ${ }^{\circledR}$ Fusion (WP-SB $562.5 \mathrm{~g} / \mathrm{kg}$ ) containing $500 \mathrm{~g} / \mathrm{kg}$ clothianidin (CTD) $+62.5 \mathrm{~g} / \mathrm{kg}$ deltamethrin (DLT), in $100 \mathrm{~g}$ water soluble bags.

\section{Methods}

\section{Preparation of the different hut trail rooms with different surfaces}

Initially, different hut rooms including cement, plaster, clay and wood surfaces selected in the appropriate village. These rooms is treated with insecticide while four untreated control containers have been maintained .

\section{Residual spraying}


Insecticide was sprayed using a compression sprayer recommended by WHO for the IRS which is equipped with a pressure gauge and HSS-8002 nozzles tips with regulator set at 24-55 PSI. Pesticide is dissolved in 10 liters of water in compression sprayer tanks. The sprayer discharge rate was set to 755 to $780 \mathrm{ml} / \mathrm{min}$. The spray duration was adjusted to spray $19 \mathrm{~m}^{2}$ in one minute. The operation is done by an expert under supervision

\section{Mosquito species tested}

Anopheles stephensi larvae have been collected from Hormoodar village $\left(27^{\circ} 19^{\prime} 14.72^{\prime \prime} \mathrm{N}, 56^{\circ} 19^{\prime} 14.80^{\prime \prime} \mathrm{E}\right)$, in the south of Bandar Abbas city during and were transferred to the insectary. The larvae is reared into F1 generation for subsequent tests.

\section{Adult susceptibility tests}

According to the guideline developed by $\mathrm{WHO}$, diagnostic dose of insecticides was used against female adult of An. stephensi mosquitoes. A total of four replicates were used as exposure (20-25 mosquitos per test, totally 100 specimens) for each insecticide and two replicates as control (totally 50 specimens). Test kits and insecticide-impregnated papers were purchased from the WHO collaborative center in University Sains Malaysia (USM), Penang, Malaysia. Bendiocarb 0.1\%, permethrin 0.75\% lambda-cyhalothrin $0.05 \%$ and deltamethrin $0.05 \%$ were used against adult mosquitoes. The control test mosquitoes were exposed to papers impregnated only with the appropriate carrier oil for pyrethroids and carbamates that was without insecticide.Tests were performed on the $F_{1}$ progeny of wild-caught adult females of filed population, 3-5 days old fed with sugar. The mosquitoes were exposed to different insecticides by 60minutes exposure time and 24 hours recovery period. A pad of a cotton wool soaked in $10 \%$ sugar solution was provided as feeding source of mosquitoes during the recovery period. Tests were carried out in an insectary maintained at $(27 \pm 2)^{\circ} \mathrm{C}$ temperature and $(75 \pm 10) \%$ relative humidity, $14: 10$ light: dark]. Mortality rate for each test was estimated at the end of tests. Results showed resistance to both pyrethroid and carbamate insecticides with mortality rates of $(84.31 \pm 2.62) \%,(89.66 \pm 2.34) \%$, $(82.27 \pm 5.20) \%$ and $(86.10 \pm 3.61) \%$ to lambda-cyhalothrin, deltamethrin, permethrin and bendiocarb, respectively. An. stephensi showed resistance to both pyrethroid and carbamate insecticide groups that used in this study (lambda-cyhalothrin, deltamethrin, permethrin, and bendiocarb).

\section{Bioassay tests}

The bioassay tests were carried out for evaluation of residual effect of insecticide by WHO cones. The cones was fitted on different treated surfaces using rubber band. About 10-12 sugar-fed, 3-5 days old female mosquitoes was gently released into each cone at the vertical position. The mosquitoes have been exposed for 30 minutes to each treated surfaces in five different replicates. The same procedures were carried out for control container. At the end of exposure time, the adults transferred into clean cups with cotton wool pad containing $10 \%$ sucrose solution and were kept in the insectary for $24 \mathrm{~h}$ recovery period, the time for recording the mortality rate. The mortality followed after 48 and 72 hours as well (WHO, 2020). (Figure. 2) 


\section{Application method:}

Minimum safety instructions and protective measures must be observed at all times. The operators will ensure that the insecticide formulation is safely and correctly applied. Standard Operating Procedures (SOPs) as well as WHO manual prepared for field staff working in national vector control programmes (WHO, 2003) applicable for Phase II trials must be available on site (e.g. safe use of compression sprayers, handling and spraying techniques, protective clothing and visors). The hut walls will be sprayed to attain dosages as per manufacturer's recommendation. The spray men will be carefully supervised during spraying. Products will be applied via Indoor Residual Spraying (IRS) with the maximum application rate amounting to $40(50) \mathrm{mL}$ spray $/ \mathrm{m}^{2}$. Only one round of spraying will be done. Calibration of the spray pumps will be done to obtain uniform and good quality spraying for the targeted dose. Protective clothing, goggles, gloves, etc. are provided to all spray men for their general safety.

Sachets and waste water will be disposed of according to WHO guidelines (WHO, 2007).

\section{Statistical analysis}

Data obtained from different replicates were collected for each surface. The mortality rate under $80 \%$ is considered as threshold level. Tests with control mortality rate between 5 and $20 \%$, were corrected using Abbott's formula.

\section{Calculation of mortality rate}

Mortality rate in plaster, mud cement, wood surfaces has been calculated and plot the diagram.

\section{Application equipment and other materials}

One Hudson ${ }^{\circledR}$ Expert compression sprayer fitted with a constant flow valve (CFV), Safety equipment: overalls, boots, helmet, visor, gloves, chemical mask.WHO testing cones (complete kit with cones and aspirators), Consumables including: cotton wool, paper cups, marker pen, masking tape, gloves, glucose, elastic bands, netting material, shoe tacks, tetramin, cerelac, filter papers, pipettes (with rubber teat), distilled water, $30 \mathrm{~cm}$ by $30 \mathrm{~cm}$ by $30 \mathrm{~cm}$ mosquito cages, mouth aspirators, stop watch, weather station (thermometers, hygrometer, etc.), tiny tags/labels, mosquitoes (charged per organism from the insectary), cool boxes and equipment to transport mosquitoes to insectary, cement,whitewash,sand, plywood/ceiling board, grass thatch, sheets galvanized iron, mud daub, insecticides, nails.

\section{Results}

Result of contact bioassay. The results of bioassay test on different surfaces are illustrated in Figures 36.

Contact bioassay tests was carried out on days $1,5,15,30,60,90,120,150,180,210,240,290$ days after application. The mortality in all surfaces until 8 months is more than $80 \%$. Mortality rate after 9 months 
on plaster and mud was more than $80 \%$. The results form cement and wood was less than $80 \%$. The cut of point of $\mathrm{WHO}$ is $80 \%$ mortality. Relative humidity and temperature of the test rooms has been recorded during the bioassay experiments.

\section{Discussion And Conclusion:}

Resistance to different insecticide classes such as pyrethroids, organophosphate, organochlorine, carbamates were evaluated using WHO guidelines. Results showed a wide variety of susceptibility/resistance status to these chemicals according to the location, historical context of pesticide used, genetic background of vectors, age and abdominal conditions of adults may play a role in the susceptibility status of these species to different insecticides. Fludora ${ }^{\circledR}$ Fusion has been developed specifically for vector control applications; it is the first product intended for indoor residual spraying campaigns which combines two unrelated modes of action, providing optimum effectiveness under conditions of insecticide resistance. The dual mode of action provides more robust and consistent residual activity compared to either active ingredient applied alone. The presence of a second mode of action helps to slow down the potential development of resistance to clothiandin. Fludora ${ }^{\circledR}$ Fusion and clothianidin WG 70 showed mortality rates over $80 \%$ WHO bio-efficacy threshold on cement walls either with susceptible or resistant $A n$. gambiae s.s. over a period of 10 and 9 months, respectively. Treatment

with Fludora ${ }^{\circledR}$ Fusion and clothianidin WG 70 on the mud walls showed residual effect for 6 months and 5 months respectively against both susceptible and resistant mosquitoes (8).Resistance of Anopheles gambiae to the new insecticide clothianidin associated with unrestricted use of agricultural neonicotinoids in Yaoundé, Cameroon has been reported (9).

\section{Declarations}

Ethics approval and consent to participate: Applicable

Consent for publication: Applicable

Availability of data and material: Applicable

Competing interests: Not applicable

Funding: Tehran University of Medical Sciences

Authors' contributions: All authors were involved

Acknowledgments: The authors would like to appreciate very much for funding of research by Institute of Environmental Research, Tehran University of Medical Sciences as well as Bayer company.

\section{References}


1. -World Health Organization. (2018). WHO recommended insecticides for indoor residual spraying against malaria vectors.

2. -World Health Organization. (2020). Global technical strategy for malaria 2016-2030. PP 35.

3. WHO. List of WHO prequalified vector control products. Geneva: World Health Organization; 2020.

4. Jeschke P, Nauen R, Beck ME. Nicotinic acetylcholine receptor agonists: a milestone for modern crop protection. Angew Chem Int Ed Engl. 2013;52(36):9464-85.

5. WHO. Global Plan for Insecticide Resistance Management in Malaria Vectors. Geneva, Switzerland: World Health Organization; 2012. https://www. who.int/malar ia/publi catio ns/atoz/gpirm /en/.

6. WHO. The technical basis for coordinated action against insecticide resistance: preserving the effectiveness of modern malaria vector control. Geneva: World Health Organization; 2010.

7. Ngufor C, Fongnikin A, Rowland M, N'Guessan R. Indoor residual spraying with a mixture of clothianidin (a neonicotinoid insecticide) and deltamethrin provides improved control and long residual activity against pyrethroid resistant Anopheles gambiae s.l. in Southern Benin. PLoS One. 2017;12:e0189575.

8. WHO. List of WHO Prequalified Vector Control Products. 2020.

9. -Fiacre R, Agossa GG, Padonou, Arsene Jacques YH, Fassinou, Esdras M, Odjo1 OK, Akuoko A, Salako ZC, Koukpo, Udoka C, Nwangwu, Bruno Akinro1, Michel Sezonlin and Martin Akogbeto C. Small-scale field evaluation of the efficacy and residual effect of Fludora ${ }^{\circledR}$ Fusion (mixture of clothianidin and deltamethrin) against susceptible and resistant Anopheles gambiae populations from Benin, West Africa. Malar J.2018; 17:484.

10. - Fouet, Ayukarah F, Ashu MM, Ambadiang W, Tchapga CS 1. Wondji and Colince Kamdem. Resistance of Anopheles gambiae to the new insecticide clothianidin associated with unrestricted use of agricultural neonicotinoids in Yaoundé, Cameroon. bioRxiv p.2020. https://www.biorxiv.org/content/10.1101/2020.08.06.239509v1.

\section{Figures}




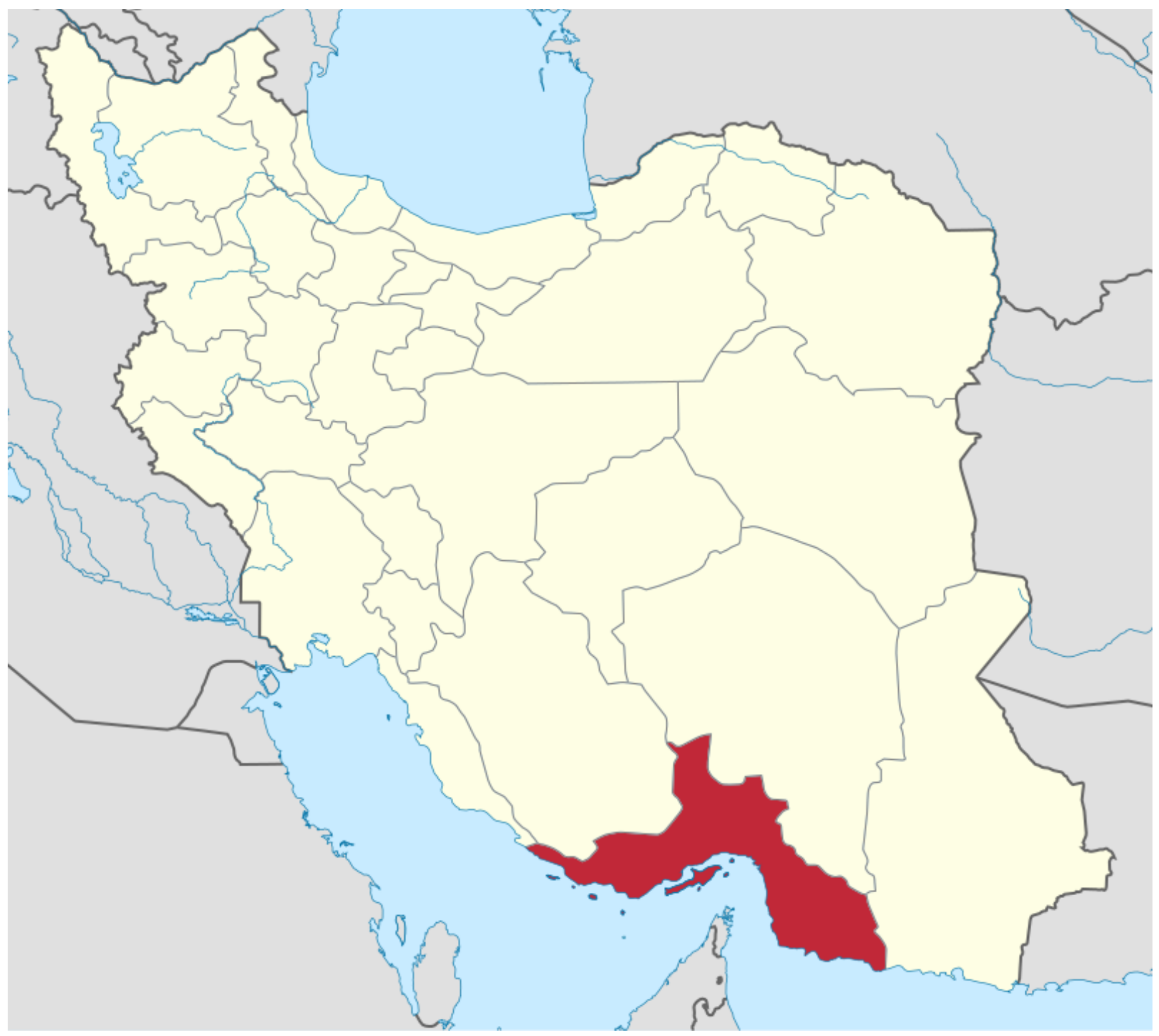

Figure 1

Map of study area, Bandar Abbas. Hormozgan Province, Southern Iran 


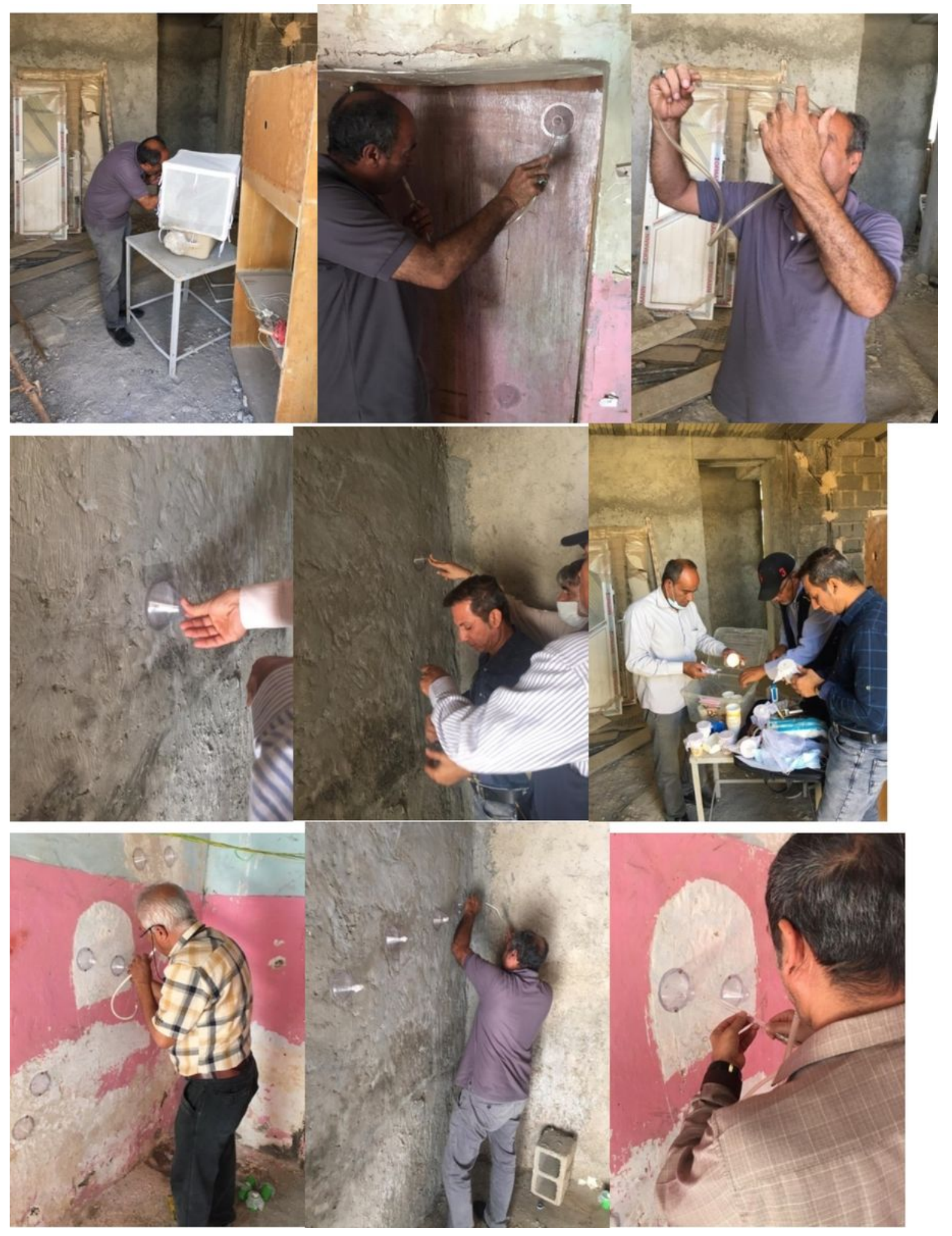

Figure 2

Evaluation of (Fludora ${ }^{\circledR}$ Fusion) on different surfaces 


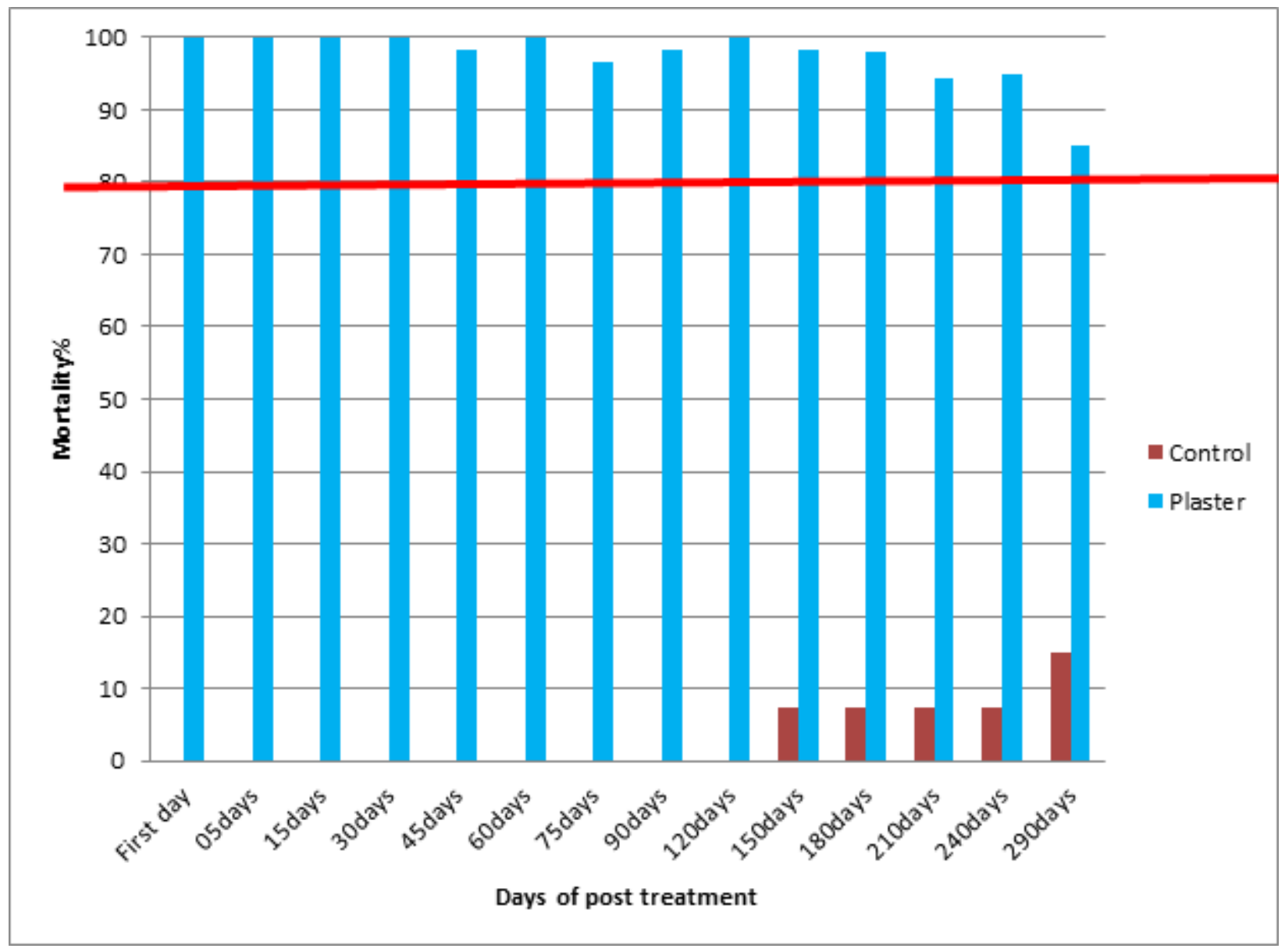

Figure 3

Residual effect of (Fludora ${ }^{\circledR}$ Fusion) on plaster surfaces 


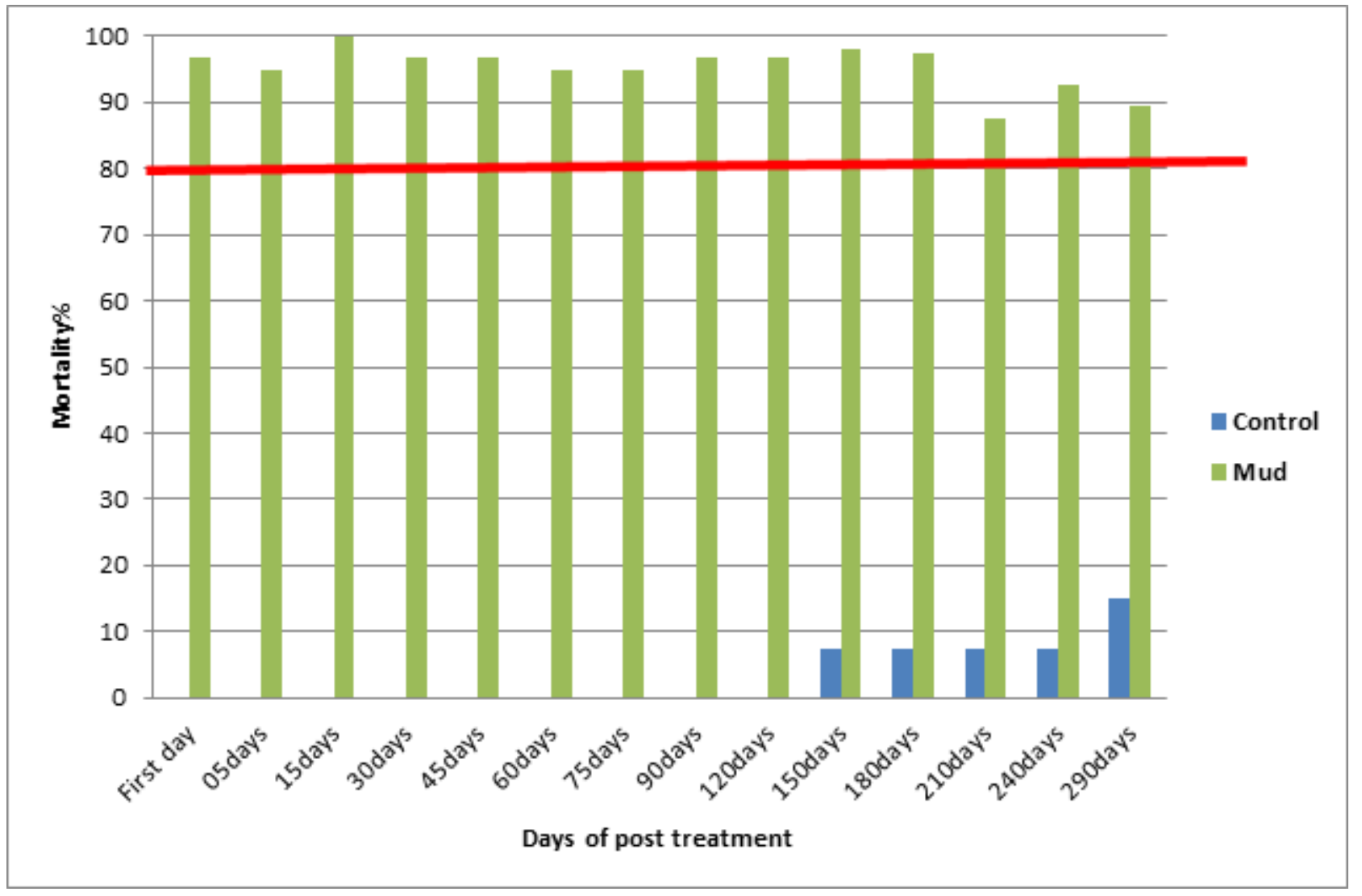

Figure 4

Residual effect of (Fludora ${ }^{\circledR}$ Fusion) on mud surfaces

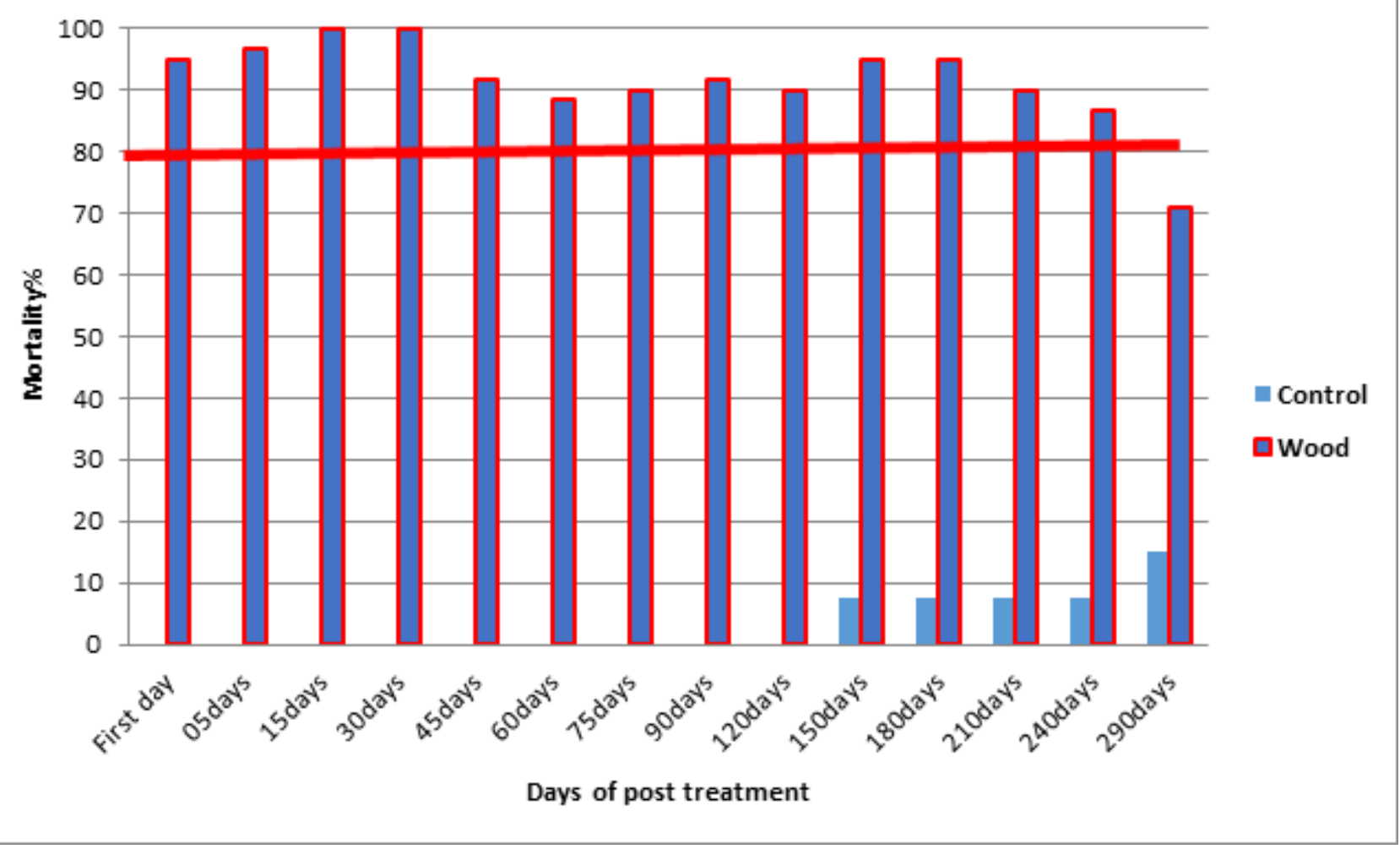

Figure 5 
Residual effect of (Fludora ${ }^{\circledR}$ Fusion) on wood surfaces

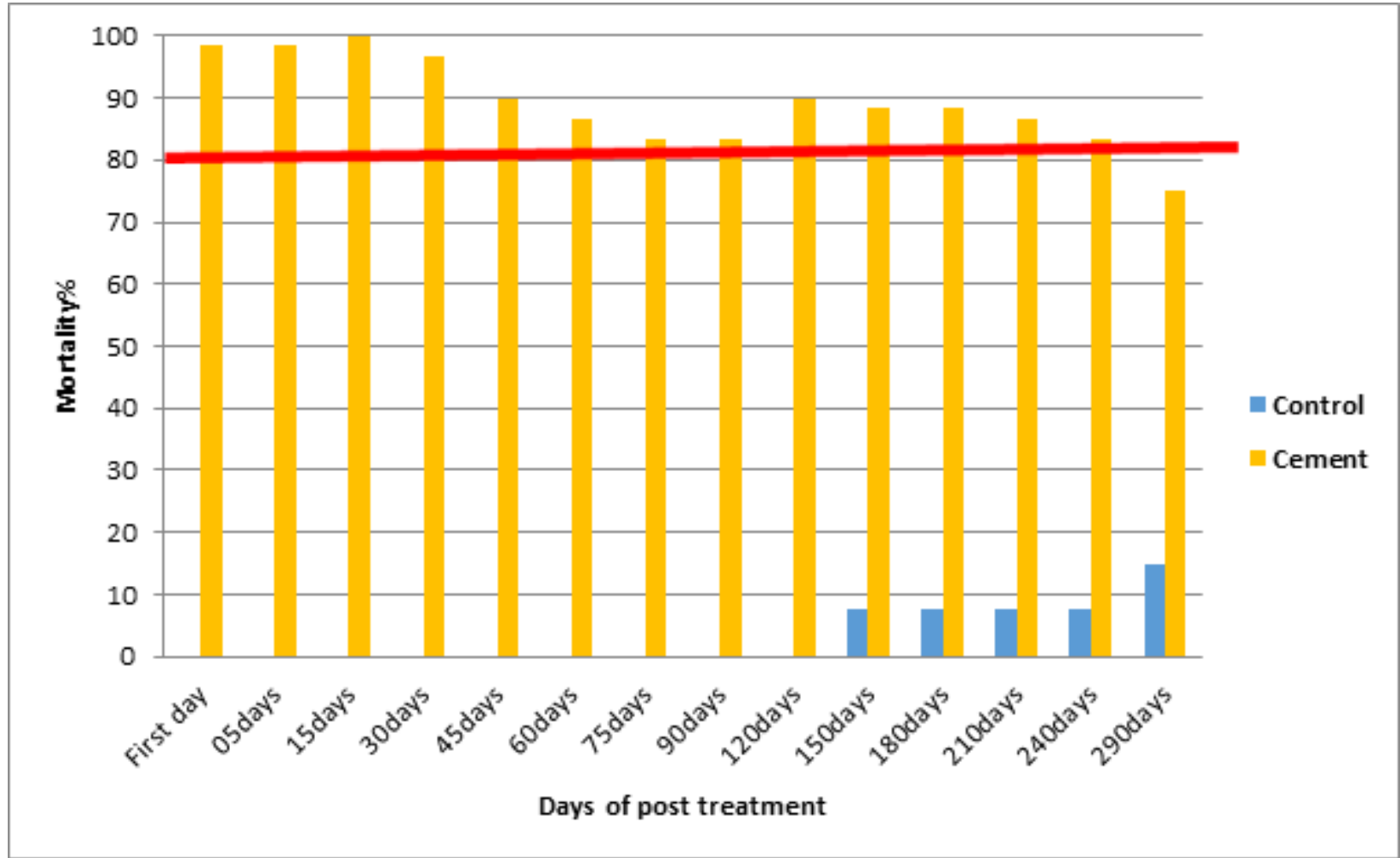

Figure 6

Residual effect of (Fludora ${ }^{\circledR}$ Fusion) in cement surfaces 